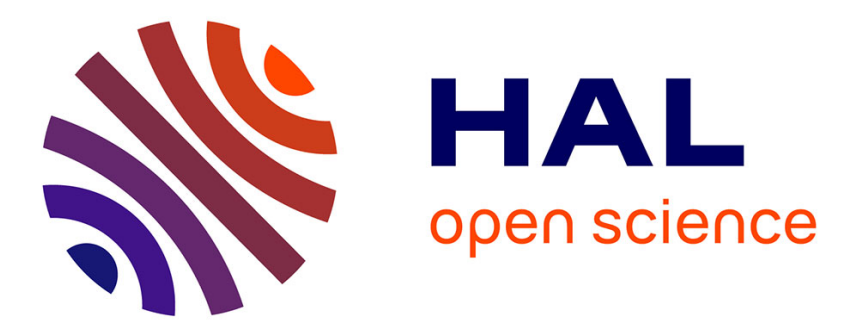

\title{
Asymptotically efficient estimators for nonparametric heteroscedastic regression models \\ Jean-Yves Brua
}

\section{To cite this version:}

Jean-Yves Brua. Asymptotically efficient estimators for nonparametric heteroscedastic regression models. Statistical Methodology, 2009, 6 (1), pp.47-60. 10.1016/j.stamet.2008.02.009 . hal-00178536v2

\section{HAL Id: hal-00178536 \\ https://hal.science/hal-00178536v2}

Submitted on 29 Nov 2007

HAL is a multi-disciplinary open access archive for the deposit and dissemination of scientific research documents, whether they are published or not. The documents may come from teaching and research institutions in France or abroad, or from public or private research centers.
L'archive ouverte pluridisciplinaire HAL, est destinée au dépôt et à la diffusion de documents scientifiques de niveau recherche, publiés ou non, émanant des établissements d'enseignement et de recherche français ou étrangers, des laboratoires publics ou privés. 


\title{
Asymptotically efficient estimators for nonparametric heteroscedastic regression models
}

\author{
J.-Y. BRUA \\ IRMA, 7 rue René Descartes F67084, Strasbourg Cedex, France
}

\begin{abstract}
This paper concerns the estimation of a function at a point in nonparametric heteroscedastic regression models with Gaussian noise or noise having unknown distribution. In those cases an asymptotically efficient kernel estimator is constructed for the minimax absolute error risk.
\end{abstract}

Key words: Asymptotical efficiency, Kernel estimator, Minimax, Nonparametric regression

$1991 M S C: 62 \mathrm{G} 08,62 \mathrm{G} 20$

\section{Introduction}

We consider the problem of estimating a regression function $S$ at a given point $\left.z_{0} \in\right] 0 ; 1[$ under observations

$$
y_{k}=S\left(x_{k}\right)+g\left(x_{k}, S\right) \xi_{k}, \quad k \in\{1, \ldots, n\}
$$

where the regressors $x_{k}=k / n$ are deterministic, $\xi_{k}$ are independent identically distributed random variables which will firstly be assumed Gaussian standard then having unknown density. Notice that the variance of the noises $g^{2}$ is unknown and depends on the unknown regression function $S$ and the regressors $x_{k}$.

Email address: brua@math.u-strasbg.fr (J.-Y. BRUA). 
Heteroscedastic regression models with this type of scale functionals have been encountered in consumer budget studies utilizing observations on individuals with diverse incomes, in analysis of investment behavior of firms of different sizes and more recently in medical research. For example, Goldfeld and Quandt (1972) considered polynomial regression models such that $y_{k}=\alpha+\beta x_{k}+$ $u_{k}, \quad \mathbb{E}\left(u_{k}^{2}\right)=a+b x_{k}+c x_{k}^{2}$, which is a particular case of our model (1) if we assume the unknown regression function being like $S(x)=\alpha+\beta x$ and $g^{2}(x, S)=\left(a-\frac{\alpha c}{\beta^{2}}\right)+\left(b-2 \frac{\alpha c}{\beta}\right) x+\frac{c}{\beta^{2}} S^{2}(x)$. Other heteroscedastic regression models are studied for instance in Efromovich and Pinsker (1996), Galtchouk and Pergamenshchikov (2005) and Efromovich (2007).

The problem of Hölder regression estimation has been studied by several authors. For a regression function belonging to a quasi-Hölder class and estimated at a point with squared error loss, Sacks and Ylvisaker (1981) showed that the linear minimax estimator is a kernel estimator. Donoho and Liv (1991) further found that this estimator is within 17 percent of asymptotically minimax among all procedures and obtained optimal kernels for Hölder classes. For estimating the whole object or its $k$ th derivative with sup-norm global loss and Hölder class, Korostelev (1993) and Donoho (1994) proved that a kernel estimator is asymptotically efficient.

This article deals with nonparametric estimation of a regression function belonging to a Hölder ball. We work with the absolute error loss and the corresponding risk. Our aim is to find an efficient estimator, that is to say an estimator which achieves the sharp asymptotic behavior of the minimax risk. To that purpose we use the method developed by Galtchouk and Pergamenshchikov (2006) who introduce the local weak Hölder classes to define the risk of an estimator. So we use the classes $\mathcal{U}_{z_{0}, \delta}$ which allows an arbitrary large derivative but has a Hölder condition based on a Hölder constant tending to zero (see $(2)$ ), then define the risk $\mathcal{R}_{z_{0}, \delta}(\tilde{S})$ of an estimator $\tilde{S}$ of $S\left(z_{0}\right)$ and the minimax risk $\inf _{\tilde{S}} \mathcal{R}_{z_{0}, \delta}(\tilde{S})$ (see $(11)$ ). In these conditions we prove that a kernel estimator is asymptotically efficient, it means that the minimax risk attains the sharp asymptotic constant.

This paper is organized as follows. In section 2 we describe the problem in the case of Gaussian noise with all assumptions needed and define all necessary mathematical objects. Our main results of this problem are written in section 3. The case of unknown noise is related in section 4 . Theorems are proved in section 5 and appendix A contains useful results for our proofs. 


\section{Statement of the problem}

Consider model $(1)$ where $g:[0 ; 1] \times C^{1}([0 ; 1], \mathbb{R}) \longrightarrow \mathbb{R}_{+}^{*}$ and $S$ are unknown functions, $S$ belonging to the class

$$
\mathcal{H}(\beta)=\bigcup_{M>0, K>0} \mathcal{H}(M, K, \beta)
$$

where $\beta=1+\alpha$ is known, $\alpha \in] 0 ; 1], \mathcal{H}(M, K, \beta)$ is the Hölder class defined as

$\mathcal{H}(M, K, \beta)=\left\{S \in C^{1}([0 ; 1], \mathbb{R}):\left\|S^{\prime}\right\| \leq M, \sup _{x, y \in[0 ; 1]} \frac{\left|S^{\prime}(y)-S^{\prime}(x)\right|}{|x-y|^{\alpha}} \leq K\right\}$,

with $\|f\|=\sup _{x \in[0 ; 1]}|f(x)|$. We suppose that the noises $\left(\xi_{k}\right)_{1 \leq k \leq n}$ are independent identically distributed $\mathcal{N}(0,1)$.

As mentioned in the introduction, we will work with a minimax risk taken over the local weak Hölder class at the point $z_{0}$ defined, for $0<\delta<1$, as

$\mathcal{U}_{z_{0}, \delta}=\left\{S \in \mathcal{H}(\beta):\left\|S^{\prime}\right\| \leq \delta^{-1} ; \forall h>0,\left|\int_{-1}^{1}\left(S\left(z_{0}+h u\right)-S\left(z_{0}\right)\right) d u\right| \leq \delta h^{\beta}\right\}$

Notice that

$$
\int_{-1}^{1}\left(S\left(z_{0}+h u\right)-S\left(z_{0}\right)\right) d u=\int_{-1}^{1}\left(\int_{z_{0}}^{z_{0}+u h}\left(S^{\prime}(t)-S^{\prime}\left(z_{0}\right)\right) d t\right) d u
$$

so we have for all $S \in \mathcal{H}(M, K, \beta)$

$$
\left|\int_{-1}^{1}\left(S\left(z_{0}+h u\right)-S\left(z_{0}\right)\right) d u\right| \leq \frac{2 K}{\beta(\beta+1)} h^{\beta} .
$$

That is why the class $\mathcal{U}_{z_{0}, \delta}$ is called a weak Hölder class.

Furthermore (3) implies that $\mathcal{H}\left(\delta^{-1}, \delta, \beta\right) \subset \mathcal{U}_{z_{0}, \delta}$ for any $0<\delta<1$.

Let us give the assumptions needed. Firstly we suppose that

$$
\lim _{n \rightarrow \infty} \sup _{S \in \mathcal{U}_{z_{0}, \delta}}\left|\left(\frac{1}{q_{n}} \sum_{k=1}^{n} Q\left(\frac{x_{k}-z_{0}}{h}\right) g^{2}\left(x_{k}, S\right)\right)^{\frac{1}{2}}-g\left(z_{0}, S\right)\right|=0,
$$

with

$$
q_{n}=\sum_{k=1}^{n} Q\left(\frac{x_{k}-z_{0}}{h}\right), \quad Q=\mathbb{I}_{[-1 ; 1]} \text { and } h=n^{-1 /(2 \beta+1)} .
$$

Moreover we assume that there exists $g_{\star}>0$ and $g^{\star}<\infty$ such that

$$
g_{\star} \leq \inf _{0 \leq x \leq 1} \inf _{S \in C^{1}([0 ; 1], \mathbb{R})} g(x, S) \leq \sup _{0 \leq x \leq 1} \sup _{S \in C^{1}([0 ; 1], \mathbb{R})} g(x, S) \leq g^{\star}
$$


and that the function $g$ is differentiable in the Frechet sense with respect to $S$ in $C^{1}([0 ; 1], \mathbb{R})$ uniformly over $x \in[0 ; 1]$, i.e. for any $S, S_{0} \in C^{1}([0 ; 1], \mathbb{R})$

$$
g(x, S)=g\left(x, S_{0}\right)+L_{x, S_{0}}\left(S-S_{0}\right)+\Gamma_{x, S_{0}}\left(S-S_{0}\right),
$$

where the linear operator $L_{x, S_{0}}$ is bounded on $C^{1}([0 ; 1], \mathbb{R})$ uniformly over $x \in[0 ; 1]$, i.e. for any $S_{0} \in C^{1}([0 ; 1], \mathbb{R})$ there exists some positive constant $C_{S_{0}}$ such that

$$
\sup _{x \in[0 ; 1]} \sup _{S \in C^{1}([0 ; 1], \mathbb{R}),\|S\| \neq 0}\left|L_{x, S_{0}}(S)\right| /\|S\| \leq C_{S_{0}}
$$

and the residual term $\Gamma_{x, S_{0}}(S)$ satisfies the property

$$
\lim _{\|S\| \rightarrow 0} \sup _{x \in[0 ; 1]} \Gamma_{x, S_{0}}(S) /\|S\|=0
$$

\section{Remark 2.1}

Note that hypothesis (4) is verified when for all $\varepsilon>0$, there exists $\eta>0$ such that if $\left|x-z_{0}\right| \leq \eta$, then $\sup _{S \in C^{1}([0 ; 1], \mathbb{R})}\left|g(x, S)-g\left(z_{0}, S\right)\right| \leq \varepsilon$.

In particular a function $g$ satisfies this property if it is uniformly continuous with respect to both variables.

\section{Remark 2.2}

Let us give a general example of a function $g$ satisfying hypothesis (4)-(8) above. Let $V: \mathbb{R} \longrightarrow \mathbb{R}_{+}$and $G:[0 ; 1] \times \mathbb{R} \longrightarrow \mathbb{R}_{+}$two differentiable functions such that

$$
\left\|V^{\prime}\right\|_{\infty}<\infty, G_{\star}=\inf _{x \in[0,1], y \in \mathbb{R}} G(x, y)>0, G_{\star}^{\prime}=\sup _{x \in[0 ; 1], y \in \mathbb{R}}\left|\frac{\partial G}{\partial y}(x, y)\right|<\infty .
$$

Define

$$
g^{2}(x, S)=G(x, S(x))+\int_{0}^{1} V(S(t)) d t .
$$

The derivative in the Frechet sense of $g$ is given by

$$
L_{x, S}(f)=\frac{1}{2 g(x, S)} \frac{\partial G}{\partial y}(x, S(x)) f(x)+\frac{1}{2 g(x, S)} \int_{0}^{1} V^{\prime}(S(t)) f(t) d t
$$

so we have

$$
\sup _{x \in[0 ; 1]} \sup _{S \in C^{1}([0 ; 1], \mathbb{R}),\|S\| \neq 0} \frac{\left|L_{x, S}(f)\right|}{\|f\|_{\infty}} \leq \frac{G_{\star}^{\prime}+\|V\|_{\infty}}{2 \sqrt{G_{\star}}} .
$$

Writing Taylor's expansion of functions $y \mapsto G(x, y)$ at the point $(x, S(x))$ and $V$ at the point $S(t)$ to the first order: 


$$
\begin{aligned}
G(x, S(x)+f(x)) & =G(x, S(x))+\frac{\partial G}{\partial y}(x, S(x)) f(x)+f(x) \varepsilon_{x, S}(f(x)), \\
V(S(t)+f(t)) & =V(S(t))+V^{\prime}(S(t)) f(t)+f(t) \tilde{\varepsilon}_{t, S}(f(t)),
\end{aligned}
$$

we can easily show that

$$
\begin{aligned}
\frac{\left|\Gamma_{x, S}(f)\right|}{\|f\|_{\infty}} \leq & \frac{G_{\star}^{\prime}+\left\|V^{\prime}\right\|_{\infty}}{8 G_{\star}^{3 / 2}}\left|g^{2}(x, S+f)-g^{2}(x, S)\right| \\
& +\frac{1}{2 \sqrt{G_{\star}}}\left(\left|\varepsilon_{x, S}(f(x))\right|+\int_{0}^{1}\left|\tilde{\varepsilon}_{t, S}(f(t))\right| d t\right) .
\end{aligned}
$$

Now if we take $G(x, y)=\alpha_{0}+\alpha_{1} x+\alpha_{2} \sin ^{2} y$ and $V(y)=\alpha_{3} \sin ^{2} y$ for all $(x, y) \in[0,1] \times \mathbb{R}$, with $\alpha_{0}>0$ and $\alpha_{1}, \alpha_{2}, \alpha_{3} \in \mathbb{R}_{+}$, then the function $g$ defined as (9) is uniformly continuous, bounded by $\sqrt{\alpha_{0}}$ and $\sqrt{\alpha_{0}+\alpha_{1}+\alpha_{2}+\alpha_{3}}$. Moreover by writing explicitly the functions $\varepsilon_{x, S}$ and $\tilde{\varepsilon}_{x, S}$ for this case, we can prove thanks to (10) that $g$ satisfies hypothesis (8). So we have exhibited an example of function $g$ which satisfies all assumptions needed.

For any estimator $\tilde{S}_{n}\left(z_{0}\right)$ of $S\left(z_{0}\right)$ we define the following risk

$$
\mathcal{R}_{z_{0}, \delta}\left(\tilde{S}_{n}\right)=\sup _{S \in \mathcal{U}_{z_{0}, \delta}} \mathbb{E}_{S} \varphi_{n} \frac{\left|\tilde{S}_{n}\left(z_{0}\right)-S\left(z_{0}\right)\right|}{g\left(z_{0}, S\right)},
$$

where $\mathbb{E}_{S}$ is the expectation taken with respect to the law $\mathbb{P}_{S}$ in (1) and $\varphi_{n}=n^{\frac{\beta}{2 \beta+1}}$.

The aim is to attain the sharp constant with this rate $\varphi_{n}$. It is only assumed that $\beta \in] 1 ; 2]$ because if $\beta>2$ we should use a kernel $Q$ of order $[\beta]$ i.e. such that $\int u^{j} Q(u) d u=0$ for $j=1,2, \ldots,[\beta]$ and $\int Q(u) d u<\infty$, where $[a]$ denotes the integer part of the number $a$.

\section{Lower and upper bounds}

In this section we give the lower bound for the minimax risk and show that the kernel estimator $\hat{S}_{n}\left(z_{0}\right)$, defined by

$$
\hat{S}_{n}\left(z_{0}\right)=\frac{1}{q_{n}} \sum_{k=1}^{n} Q\left(\frac{x_{k}-z_{0}}{h}\right) y_{k}
$$

is asymptotically efficient as we give the upper bound for its risk.

Theorem 3.1 For any $\delta \in] 0 ; 1[$,

$$
\liminf _{n \rightarrow \infty} \inf _{\tilde{S}} \mathcal{R}_{z_{0}, \delta}(\tilde{S}) \geq \frac{\mathbb{E}|\xi|}{\sqrt{2}}, \quad \xi \sim \mathcal{N}(0,1)
$$


where the infimum is taken over all estimators $\tilde{S}$ of $S\left(z_{0}\right)$.

Theorem 3.2 For the estimator $\hat{S}_{n}\left(z_{0}\right)$ from (12), the following inequality holds:

$$
\limsup _{\delta \rightarrow 0} \limsup _{n \rightarrow \infty} \mathcal{R}_{z_{0}, \delta}\left(\hat{S}_{n}\left(z_{0}\right)\right) \leq \frac{\mathbb{E}|\xi|}{\sqrt{2}}, \quad \xi \sim \mathcal{N}(0,1)
$$

\section{Case of unknown noise distribution}

In this section we suppose that the $\left(\xi_{k}\right)$ in model $(1)$ are independent identically distributed with an unknown density $p$ belonging to

$$
\mathcal{P}_{\varepsilon, L}=\left\{p: \int_{-\infty}^{+\infty} x p(x) d x=0, \int_{-\infty}^{+\infty} x^{2} p(x) d x=1, \int_{-\infty}^{+\infty}|x|^{2+\varepsilon} p(x) d x \leq L\right\}
$$

with $\varepsilon>0$ and $L>0$ sufficiently large to have the density of the standard Gaussian random variable in $\mathcal{P}_{\varepsilon, L}$.

We define the risk corresponding to this case as

$$
\tilde{\mathcal{R}}_{z_{0}, \delta}\left(\tilde{S}_{n}\right)=\sup _{p \in \mathcal{P}_{\epsilon, L}} \sup _{S \in \mathcal{U}_{z_{0}, \delta}} \mathbb{E}_{S} \varphi_{n} \frac{\left|\tilde{S}_{n}\left(z_{0}\right)-S\left(z_{0}\right)\right|}{g\left(z_{0}, S\right)}
$$

In the following theorems we give the sharp lower bound for the minimax risk over all estimators and establish the upper bound for the minimax risk for the kernel estimator $\hat{S}_{n}\left(z_{0}\right)$ of $S\left(z_{0}\right)$ defined in (12).

Theorem 4.1 For any $\delta \in] 0 ; 1[$, one has:

$$
\liminf _{n \rightarrow \infty} \inf _{\tilde{S}} \tilde{\mathcal{R}}_{z_{0}, \delta}(\tilde{S}) \geq \frac{\mathbb{E}|\eta|}{\sqrt{2}}, \quad \eta \sim \mathcal{N}(0,1)
$$

where the infimum is taken over all estimators $\tilde{S}$ of $S\left(z_{0}\right)$.

Theorem 4.2 The kernel estimator (12) is asymptotically efficient. Indeed it satisfies the inequality:

$$
\limsup _{\delta \rightarrow 0} \limsup _{n \rightarrow \infty} \tilde{\mathcal{R}}_{z_{0}, \delta}\left(\hat{S}_{n}\left(z_{0}\right)\right) \leq \frac{\mathbb{E}|\eta|}{\sqrt{2}}, \quad \eta \sim \mathcal{N}(0,1)
$$




\section{Proof of the theorems}

\subsection{Proof of theorem 3.1}

For all $\nu \in] 0 ; \frac{1}{4}\left[\right.$, denote $S_{\nu}(x)=\varphi_{n}^{-1} V_{\nu}\left(\frac{x-z_{0}}{h}\right)$, where the function $V_{\nu}$ is defined by:

$$
V_{\nu}(x)=\frac{1}{\nu} \int_{-\infty}^{+\infty} \tilde{Q}_{\nu}(u) l\left(\frac{u-x}{\nu}\right) d u, \tilde{Q}_{\nu}(u)=\mathbb{I}_{\{|u| \leq 1-2 \nu\}}+2 \mathbb{I}_{\{1-2 \nu \leq|u| \leq 1-\nu\}},
$$

and $l$ is a non-negative function, infinitely differentiable on $\mathbb{R}$, such that for $|z| \geq 1, l(z)=0$ and $\int_{-1}^{1} l(z) d z=1$. One can easily see that for any $0<\nu<\frac{1}{4}$, we have $V_{\nu}(0)=1$ and $\int_{-1}^{1} V_{\nu}(x) d x=2$.

Let $\nu \in] 0 ; \frac{1}{4}[, b>0$ and $\delta \in] 0 ; 1\left[\right.$. Denote $S_{\nu, u}(x)=\frac{u}{\varphi_{n}} V_{\nu}\left(\frac{x-z_{0}}{h}\right)$, where $x, u \in \mathbb{R}$.

Thanks to lemma A.1, if $|u| \leq b$ there exists an integer $n_{\nu, b, \delta}>0$ such that $S_{\nu, u} \in \mathcal{U}_{z_{0}, \delta}$ for all $n \geq n_{\nu, b, \delta}$. Therefore for $n \geq n_{\nu, b, \delta}$, one has:

$$
\begin{aligned}
\mathcal{R}_{z_{0}, \delta}(\tilde{S}) & \geq \sup _{|u| \leq b} \frac{1}{g\left(z_{0}, S_{\nu, u}\right)} \mathbb{E}_{S_{\nu, u}} \varphi_{n}\left|\tilde{S}\left(z_{0}\right)-S_{\nu, u}\left(z_{0}\right)\right| \\
& \geq \frac{1}{2 b} \int_{-b}^{b} \frac{1}{g\left(z_{0}, S_{\nu, u}\right)} \mathbb{E}_{S_{\nu, u}} v_{a}\left(\varphi_{n}\left(\tilde{S}\left(z_{0}\right)-S_{\nu, u}\left(z_{0}\right)\right)\right) d u:=I_{n}(a, b),
\end{aligned}
$$

where $v_{a}(x)=|x| \wedge a, a>0$.

Write $\mathbb{P}_{S_{\nu, u}}$ the law of $\left(y_{k}^{(1)}\right)_{k=1, \ldots, n}$, where $y_{k}^{(1)}=S_{\nu, u}\left(x_{k}\right)+g\left(x_{k}, S_{\nu, u}\right) \xi_{k}$, and $\mathbb{P}$ the law of $\left(y_{k}^{(0)}\right)_{k=1, \ldots, n}$, where $y_{k}^{(0)}=g\left(x_{k}, S_{\nu, u}\right) \xi_{k}$. These two measures are equivalent and the corresponding Radon-Nikodym derivative is at the point $\left(y_{1}, \ldots, y_{n}\right)$ :

$$
\begin{aligned}
\rho_{n}(u) & =\frac{d \mathbb{P}_{S_{\nu, u}}}{d \mathbb{P}}\left(y_{1}, \ldots, y_{n}\right) \\
& =\exp \left\{-\frac{1}{2} \sum_{k=1}^{n}\left(\left(\frac{y_{k}-S_{\nu, u}\left(x_{k}\right)}{g\left(x_{k}, S_{\nu, u}\right)}\right)^{2}-\left(\frac{y_{k}}{g\left(x_{k}, S_{\nu, u}\right)}\right)^{2}\right)\right\} \\
& =\exp \left(u \varsigma_{n} \eta_{n}-\frac{u^{2}}{2} \varsigma_{n}^{2}\right)
\end{aligned}
$$


where $\varsigma_{n}^{2}=\frac{1}{\varphi_{n}^{2}} \sum_{k=1}^{n} \frac{V_{\nu}^{2}\left(\frac{x_{k}-z_{0}}{h}\right)}{g^{2}\left(x_{k}, S_{\nu, u}\right)}$ and $\eta_{n}=\frac{1}{\varsigma_{n} \varphi_{n}} \sum_{k=1}^{n} \frac{V_{\nu}\left(\frac{x_{k}-z_{0}}{h}\right)}{g^{2}\left(x_{k}, S_{\nu, u}\right)} y_{k}$.

Under the law $\mathbb{P}, \eta_{n}$ is a standard Gaussian random variable.

We prove in lemma A.2 that

$$
\varsigma_{n}^{2} \underset{n \rightarrow \infty}{\longrightarrow} \int_{-1}^{1} \frac{V_{\nu}^{2}(z)}{g^{2}\left(z_{0}, 0\right)} d z=: \sigma_{\nu}^{2}
$$

So we rewrite $\rho_{n}(u)=\exp \left(u \sigma_{\nu} \eta_{n}-\frac{u^{2} \sigma_{\nu}^{2}}{2}+r_{n}\right)$, where $r_{n}$ converges in $\mathbb{P}_{-}$ probability to zero.

Denoting $\psi_{a, n}\left(\tilde{S}, S_{\nu, u}\right)=v_{a}\left(\varphi_{n}\left(\tilde{S}_{n}\left(z_{0}\right)-S_{\nu, u}\left(z_{0}\right)\right)\right)$ and $\mathbb{E}$ the expectation for the probability measure $\mathbb{P}$, one has

$$
I_{n}(a, b) \geq \frac{1}{2 b} \int_{-b}^{b} \mathbb{E} \mathbb{I}_{B_{d}} \frac{\psi_{a, n}\left(\tilde{S}, S_{\nu, u}\right)}{g\left(z_{0}, S_{\nu, u}\right)} \varrho_{n}(u) d u+\delta_{n}(a, b)=: J_{n}(a, b)+\delta_{n}(a, b),
$$

where

$$
\begin{aligned}
B_{d} & =\left\{\left|\eta_{n}\right| \leq d\right\} \text { and } d=\sigma_{\nu}(b-\sqrt{b}), b>1, \\
\varrho_{n}(u) & =\exp \left(u \sigma_{\nu} \eta_{n}-\frac{u^{2} \sigma_{\nu}^{2}}{2}\right), \\
\delta_{n}(a, b) & =\frac{1}{2 b} \int_{-b}^{b} \mathbb{E} \mathbb{I}_{B_{d}} \frac{\psi_{a, n}\left(\tilde{S}, S_{\nu, u}\right)}{g\left(z_{0}, S_{\nu, u}\right)} \theta_{n}(u) d u, \\
\theta_{n}(u) & =\rho_{n}(u)-\varrho_{n}(u) .
\end{aligned}
$$

Note that $\rho_{n}(u) \underset{n \rightarrow \infty}{\stackrel{\mathcal{L}}{\longrightarrow}} \rho_{\infty}(u)=\exp \left(u \sigma_{\nu} \eta-\frac{u^{2} \sigma_{\nu}^{2}}{2}\right)$. We can easily show that $\mathbb{E} \rho_{\infty}(u)=1$ and we have also $\mathbb{E} \rho_{n}(u)=1$ because $\rho_{n}(u)$ is a density. Hence, using theorem 3.6 from Billingsley (1999), $\left\{\rho_{n}(u), n \geq 1\right\}$ is uniformly integrable. And since $\varrho_{n}(u)$ is bounded on $B_{d}$, we obtain the uniform integrability of $\left\{\mathbb{I}_{B_{d}} \psi_{a, n}\left(\tilde{S}, S_{\nu, u}\right) \theta_{n}(u), n \geq 1\right\}$.

Write $\theta_{n}(u)=\exp \left(u \sigma_{\nu} \eta_{n}-\frac{u^{2} \sigma_{\nu}^{2}}{2}\right)\left(e^{r_{n}}-1\right)$ and notice that $\exp \left(u \sigma_{\nu} \eta_{n}-\frac{u^{2} \sigma_{\nu}^{2}}{2}\right)$ is bounded on $B_{d}$ and that $e^{r_{n}}-1 \underset{n \rightarrow \infty}{\stackrel{\mathbb{P}}{\longrightarrow}} 0$. As a consequence one has

$$
\frac{\mathbb{I}_{B_{d}} \psi_{a, n}\left(\tilde{S}, S_{\nu, u}\right)}{g\left(z_{0}, S_{\nu, u}\right)} \theta_{n}(u) \underset{n \rightarrow \infty}{\stackrel{\mathbb{P}}{\longrightarrow}} 0
$$

It follows that $\frac{\mathbb{I}_{B_{d}} \psi_{a, n}\left(\tilde{S}, S_{\nu, u}\right)}{g\left(z_{0}, S_{\nu, u}\right)} \theta_{n}(u) \underset{n \rightarrow \infty}{\stackrel{\mathbb{L}^{1}}{\longrightarrow}} 0$ and $\mathbb{E} \frac{\mathbb{I}_{B_{d}} \psi_{a, n}\left(\tilde{S}, S_{\nu, u}\right)}{g\left(z_{0}, S_{\nu, u}\right)} \theta_{n}(u) \underset{n \rightarrow \infty}{\longrightarrow} 0$.

Finally bounded convergence yields $\delta_{n}(a, b) \underset{n \rightarrow \infty}{\longrightarrow} 0$ in (14).

Now we are interested in the term $J_{n}(a, b)$ in (14). 
First rewrite $\varrho_{n}(u)=\zeta_{n} e^{-\sigma_{\nu}^{2}\left(u-\tilde{\eta}_{n}\right)^{2} / 2}$ with $\zeta_{n}=e^{\eta_{n}^{2} / 2}$ and $\tilde{\eta}_{n}=\frac{\eta_{n}}{\sigma_{\nu}}$. Then if $\xi \sim \mathcal{N}(0,1)$ denote $\tilde{\xi}=\frac{\xi}{\sigma_{\nu}}, \zeta=e^{\xi^{2} / 2}, \tilde{B}_{d}=\{|\xi| \leq d\}$ and $\tilde{\mathbb{E}}$ the expectation for the probability law of $\xi$. With $t_{n}=\varphi_{n} \tilde{S}_{n}\left(z_{0}\right)$, we get

$$
\begin{aligned}
J_{n}(a, b) & =\frac{1}{2 b} \int_{-b}^{b} \mathbb{E}_{B_{d}} \zeta \frac{v_{a}\left(u-t_{n}\right)}{g\left(z_{0}, S_{\nu, u}\right)} \exp \left(-\frac{\sigma_{\nu}^{2}}{2}\left(u-\tilde{\eta}_{n}\right)^{2}\right) d u \\
& =\frac{1}{2 b} \int_{-b}^{b} \tilde{\mathbb{E}}_{\tilde{B}_{d}} \zeta \frac{v_{a}\left(u-t_{n}\right)}{g\left(z_{0}, S_{\nu, u}\right)} \exp \left(-\frac{\sigma_{\nu}^{2}}{2}(u-\tilde{\xi})^{2}\right) d u \\
& =\tilde{\mathbb{E}} \mathbb{I}_{\tilde{B}_{d}} \zeta \frac{1}{2 b} \int_{-b}^{b} \frac{v_{a}\left(u-t_{n}\right)}{g\left(z_{0}, S_{\nu, u}\right)} \exp \left(-\frac{\sigma_{\nu}^{2}}{2}(u-\tilde{\xi})^{2}\right) d u
\end{aligned}
$$

We have the following limit

$\tilde{\mathbb{E}} \mathbb{I}_{\tilde{B}_{d}} \zeta \frac{1}{2 b} \int_{-b}^{b} v_{a}\left(u-t_{n}\right) \exp \left(-\frac{\sigma_{\nu}^{2}}{2}(u-\tilde{\xi})^{2}\right)\left(\frac{1}{g\left(z_{0}, S_{\nu, u}\right)}-\frac{1}{g\left(z_{0}, 0\right)}\right) d u \underset{n \rightarrow \infty}{\longrightarrow} 0$.

Indeed, using hypothesis (6) and (7) one obtains

$$
\begin{aligned}
& \left|\tilde{\mathbb{E}}_{\tilde{B}_{d}} \zeta \frac{1}{2 b} \int_{-b}^{b} v_{a}\left(u-t_{n}\right) \exp \left(-\frac{\sigma_{\nu}^{2}}{2}(u-\tilde{\xi})^{2}\right)\left(\frac{1}{g\left(z_{0}, S_{\nu, u}\right)}-\frac{1}{g\left(z_{0}, 0\right)}\right) d u\right| \\
\leq & \tilde{\mathbb{E}}_{\tilde{B}_{d}} \zeta \frac{1}{2 b} \int_{-b}^{b} v_{a}\left(u-t_{n}\right) \exp \left(-\frac{\sigma_{\nu}^{2}}{2}(u-\tilde{\xi})^{2}\right)\left|\frac{\Gamma_{z_{0}, 0}\left(S_{\nu, u}\right)-L_{z_{0}, 0}\left(S_{\nu, u}\right)}{g_{\star}^{2}}\right| d u \\
\leq & \tilde{\mathbb{E}}_{\tilde{B}_{d}} \zeta \frac{1}{2 b} \int_{-b}^{b} a \frac{C_{0}\left\|S_{\nu, u}\right\|+\left|\Gamma_{z_{0}, 0}\left(S_{\nu, u}\right)\right|}{g_{\star}^{2}} d u .
\end{aligned}
$$

Since $\left\|S_{\nu, u}\right\|$ tends to zero as $n$ goes to infinity, hypothesis (8) and (15) allows then us to say that

$$
\liminf _{n \rightarrow \infty} J_{n}(a, b)=\liminf _{n \rightarrow \infty} \tilde{\mathbb{E}}_{\tilde{B}_{d}} \zeta \frac{1}{2 b} \int_{-b}^{b} \frac{v_{a}\left(u-t_{n}\right)}{g\left(z_{0}, 0\right)} \exp \left(-\frac{\sigma_{\nu}^{2}}{2}(u-\tilde{\xi})^{2}\right) d u
$$

But

$$
\begin{aligned}
& \tilde{\mathbb{E}}_{\tilde{B}_{d}} \zeta \frac{1}{2 b} \int_{-b}^{b} \frac{v_{a}\left(u-t_{n}\right)}{g\left(z_{0}, 0\right)} \exp \left(-\frac{\sigma_{\nu}^{2}}{2}(u-\tilde{\xi})^{2}\right) d u \\
\geq & \tilde{\mathbb{E}} \mathbb{I}_{\tilde{B}_{d}} \zeta \frac{1}{2 b} \int_{-\sqrt{b}}^{\sqrt{b}} \frac{v_{a}\left(t-t_{n}+\tilde{\xi}\right)}{g\left(z_{0}, 0\right)} \exp \left(-\frac{\sigma_{\nu}^{2}}{2} t^{2}\right) d t \\
\geq & \tilde{\mathbb{E}}_{\tilde{B}_{d}} \zeta \frac{1}{2 b} \int_{-\sqrt{b}}^{\sqrt{b}} \frac{v_{a}(t)}{g\left(z_{0}, 0\right)} \exp \left(-\frac{\sigma_{\nu}^{2}}{2} t^{2}\right) d t,
\end{aligned}
$$


this last inequality holds thanks to Anderson's lemma (see Ibragimov and Has'minskii, 1981, Chapter II, Lemma 10.1 and Corollary 10.2).

Eventually using the fact that $\tilde{\mathbb{E}}_{\tilde{B}_{d}} \zeta=\frac{2 \sigma_{\nu}(b-\sqrt{b})}{\sqrt{2 \pi}}$ it follows that

$$
\liminf _{a \rightarrow \infty} \liminf _{n \rightarrow \infty} J_{n}(a, b) \geq \frac{\sigma_{\nu}}{\sqrt{2 \pi}} \frac{b-\sqrt{b}}{b} \int_{-\sqrt{b}}^{\sqrt{b}} \frac{|t|}{g\left(z_{0}, 0\right)} \exp \left(-\frac{\sigma_{\nu}^{2}}{2} t^{2}\right) d t
$$

We complete the proof limiting successively $b \rightarrow \infty, \nu \rightarrow 0$ and utilizing $\sigma_{\nu}^{2} \underset{\nu \rightarrow 0}{\longrightarrow} \frac{2}{g^{2}\left(z_{0}, 0\right)}$

\subsection{Proof of theorem 3.2}

We begin by rewriting the kernel estimator as $\hat{S}_{n}\left(z_{0}\right)-S\left(z_{0}\right)=B_{n}+\frac{1}{\sqrt{q_{n}}} \zeta_{n}$ with

$$
\begin{aligned}
B_{n} & =\frac{1}{q_{n}} \sum_{k=1}^{n} Q\left(\frac{x_{k}-z_{0}}{h}\right)\left(S\left(x_{k}\right)-S\left(z_{0}\right)\right) \\
\zeta_{n} & =\frac{1}{\sqrt{q_{n}}} \sum_{k=1}^{n} Q\left(\frac{x_{k}-z_{0}}{h}\right) g\left(x_{k}, S\right) \xi_{k} .
\end{aligned}
$$

First we take a look at the term $\frac{\zeta_{n}}{\sqrt{q_{n}}}$. By $(17), \zeta_{n}$ is a Gaussian random variable $\mathcal{N}\left(0, \sigma_{n}^{2}(S)\right)$ where $\sigma_{n}^{2}(S)=\frac{1}{q_{n}} \sum_{k=1}^{n} Q\left(\frac{x_{k}-z_{0}}{h}\right) g^{2}\left(x_{k}, S\right)$. We prove in lemma A.3 that the variance $\sigma_{n}^{2}(S)$ satisfies $\sigma_{n}^{2}(S) \underset{n \rightarrow \infty}{\longrightarrow} g^{2}\left(z_{0}, S\right)$. If $\xi \sim \mathcal{N}(0,1)$, one has

$$
\begin{aligned}
\sup _{S \in \mathcal{U}_{z_{0}, \delta}} \frac{1}{g\left(z_{0}, S\right)} \mathbb{E}_{S}\left|\frac{\varphi_{n}}{\sqrt{q_{n}}} \zeta_{n}\right| & =\frac{\varphi_{n}}{\sqrt{q_{n}}} \mathbb{E}|\xi| \sup _{S \in \mathcal{U}_{z_{0}, \delta}} \frac{\sigma_{n}(S)}{g\left(z_{0}, S\right)} \\
& \leq \frac{\varphi_{n}}{\sqrt{q_{n}}} \frac{\mathbb{E}|\xi|}{g_{\star}}\left(\sup _{S \in \mathcal{U}_{z_{0}, \delta}}\left|\sigma_{n}(S)-g\left(z_{0}, S\right)\right|+g_{\star}\right) .
\end{aligned}
$$

According to hypothesis (4) and since $\frac{q_{n}}{\varphi_{n}^{2}}=\frac{q_{n}}{n h} \underset{n \rightarrow \infty}{\longrightarrow}$, we obtain

$$
\limsup _{n \rightarrow \infty} \sup _{S \in \mathcal{U} z_{0}, \delta} \mathbb{E}_{S} \frac{\varphi_{n}}{g\left(z_{0}, S\right)} \frac{\left|\zeta_{n}\right|}{\sqrt{q_{n}}} \leq \frac{\mathbb{E}|\xi|}{\sqrt{2}}
$$


Now denote $u_{k}=\frac{x_{k}-z_{0}}{h}, \Delta u_{k}=\frac{1}{n h}$ and rewrite (16) as

$$
\begin{aligned}
B_{n} & =\frac{\varphi_{n}^{2}}{q_{n}} \sum_{k=1}^{n} Q\left(u_{k}\right)\left(S\left(z_{0}+h u_{k}\right)-S\left(z_{0}\right)\right) \Delta u_{k} \\
& =\frac{\varphi_{n}^{2}}{q_{n}} \int_{-1}^{1}\left(S\left(z_{0}+h u\right)-S\left(z_{0}\right)\right) d u+\frac{\varphi_{n}^{2}}{q_{n}} R_{n}
\end{aligned}
$$

with

$$
\begin{aligned}
R_{n} & =\sum_{k=1}^{n} Q\left(u_{k}\right)\left(S\left(z_{0}+h u_{k}\right)-S\left(z_{0}\right)\right) \Delta u_{k}-\int_{-1}^{1}\left(S\left(z_{0}+h u\right)-S\left(z_{0}\right)\right) d u \\
& =\sum_{k=k_{*}}^{k^{*}} \int_{u_{k-1}}^{u_{k}}\left(S\left(z_{0}+h u_{k}\right)-S\left(z_{0}+h u\right)\right) d u \\
& -\int_{u_{k^{*}}}^{1}\left(S\left(z_{0}+h u\right)-S\left(z_{0}\right)\right) d u+\int_{u_{k_{*}-1}}^{-1}\left(S\left(z_{0}+h u\right)-S\left(z_{0}\right)\right) d u
\end{aligned}
$$

where $k^{*}=\left[n\left(z_{0}+h\right)\right]$ et $k_{*}=\left[n\left(z_{0}-h\right)\right]+1$.

We can bound $R_{n}$ as follows:

$$
\begin{aligned}
\left|R_{n}\right| & \leq \sum_{k=k_{*}}^{k^{*}} \int_{u_{k-1}}^{u_{k}} h\left(u_{k}-u\right) \delta^{-1} d u+\int_{u_{k^{*}}}^{1} h \delta^{-1} u d u+\int_{u_{k_{*}-1}}^{-1} h \delta^{-1}|u| d u \\
& \leq h \delta^{-1}\left(\sum_{k=k_{*}}^{k^{*}}\left(u_{k}-u_{k-1}\right) \frac{1}{n h}+\left(1-u_{k^{*}}\right)+2\left(-1-u_{k_{*}-1}\right)\right) \leq \frac{6 \delta^{-1}}{n} .
\end{aligned}
$$

Hence

$$
\limsup _{n \rightarrow \infty} \sup _{S \in \mathcal{U}_{z_{0}, \delta}} \mathbb{E}_{S} \varphi_{n}\left|\frac{\varphi_{n}^{2}}{q_{n}} R_{n}\right|=0
$$

With regard to the term $\frac{\varphi_{n}^{2}}{q_{n}} \int_{-1}^{1}\left(S\left(z_{0}+h u\right)-S\left(z_{0}\right)\right) d u$ in $(20)$ one has

$$
\left|\frac{\varphi_{n}^{2}}{q_{n}} \int_{-1}^{1}\left(S\left(z_{0}+h u\right)-S\left(z_{0}\right)\right) d u\right| \leq \frac{\varphi_{n}^{2}}{q_{n}} \delta n^{\frac{-\beta}{2 \beta+1}}=\delta \frac{\varphi_{n}}{q_{n}} .
$$

Then using the definition of $\mathcal{U}_{z_{0}, \delta}$ we get

$$
\limsup _{n \rightarrow \infty} \sup _{S \in \mathcal{U}_{z_{0}, \delta}} \mathbb{E}_{S} \varphi_{n}\left|\frac{\varphi_{n}^{2}}{q_{n}} \int_{-1}^{1}\left(S\left(z_{0}+h u\right)-S\left(z_{0}\right)\right) d u\right| \leq \frac{\delta}{2} .
$$


Finally (18), (21) and limiting $\delta \rightarrow 0$ in (22) yield

$$
\limsup _{\delta \rightarrow 0} \limsup _{n \rightarrow \infty} \mathcal{R}_{z_{0}, \delta}\left(\hat{S}_{n}\left(z_{0}\right)\right) \leq \frac{\mathbb{E}|\xi|}{\sqrt{2}}
$$

\subsection{Proof of theorem 4.1}

This is a consequence of the theorem 3.1 which gives the sharp lower bound in the case of Gaussian errors having expectation zero and unknown variance which depends on the design point and the regression function. The corresponding risk $\mathcal{R}_{z_{0}, \delta}$ is less than the risk $\tilde{\mathcal{R}}_{z_{0}, \delta}$ because the density of the standard Gaussian random variable belongs to $\mathcal{P}_{\varepsilon, L}$. The inequality in theorem 4.1 is then proved.

\subsection{Proof of theorem 4.2}

Writing $\hat{S}_{n}\left(z_{0}\right)-S\left(z_{0}\right)=B_{n}+\zeta_{n} / \sqrt{q_{n}}$, with $B_{n}$ and $\zeta_{n}$ defined by (16) and (17), we remark that $B_{n}$ does not depend on the distributions of the random variables $\xi_{k}$. That is the reason why (21) and (22) remain available and provide for any $\delta \in] 0 ; 1[$ :

$$
\limsup _{n \rightarrow \infty} \sup _{S \in \mathcal{U}_{z_{0}, \delta}} \varphi_{n}\left|B_{n}\right| \leq \delta / 2 .
$$

Hence it suffices to prove that

$$
\lim _{n \rightarrow \infty} \sup _{p \in \mathcal{P}_{\epsilon, L}} \sup _{S \in \mathcal{U}_{z_{0}, \delta}}\left|\frac{\mathbb{E}_{S}\left|\zeta_{n}\right|}{g\left(z_{0}, S\right)}-\mathbb{E}\right| \eta||=0
$$

with $\eta \sim \mathcal{N}(0,1)$.

Denote $\tilde{\zeta}_{n}=\zeta_{n} / g\left(z_{0}, S\right)=\sum_{k=1}^{n} u_{k}$, where $u_{k}=\frac{1}{\sqrt{q_{n}}} Q\left(\frac{x_{k}-z_{0}}{h}\right) \frac{g\left(x_{k}, S\right)}{g\left(z_{0}, S\right)} \xi_{k}$, and rewrite $\frac{g\left(x_{k}, S\right)}{g\left(z_{0}, S\right)} \xi_{k}=\xi_{k}^{\prime}+\xi_{k}^{\prime \prime}$, where

$$
\begin{aligned}
& \xi_{k}^{\prime}=\frac{g\left(x_{k}, S\right)}{g\left(z_{0}, S\right)} \xi_{k} \mathbb{I}_{\left|\xi_{k}\right| \leq q_{n}^{1 / 4}}-\frac{g\left(x_{k}, S\right)}{g\left(z_{0}, S\right)} \mathbb{E}\left(\xi_{1} \mathbb{I}_{\left|\xi_{1}\right| \leq q_{n}^{1 / 4}}\right), \\
& \xi_{k}^{\prime \prime}=\frac{g\left(x_{k}, S\right)}{g\left(z_{0}, S\right)} \xi_{k} \mathbb{I}_{\left|\xi_{k}\right|>q_{n}^{1 / 4}}-\frac{g\left(x_{k}, S\right)}{g\left(z_{0}, S\right)} \mathbb{E}\left(\xi_{1} \mathbb{I}_{\left|\xi_{1}\right|>q_{n}^{1 / 4}}\right) .
\end{aligned}
$$


Let $u_{k}^{\prime}=\frac{1}{\sqrt{q_{n}}} Q\left(\frac{x_{k}-z_{0}}{h}\right) \xi_{k}^{\prime}$ and $u_{k}^{\prime \prime}=\frac{1}{\sqrt{q_{n}}} Q\left(\frac{x_{k}-z_{0}}{h}\right) \xi_{k}^{\prime \prime}$, then one gets $\tilde{\zeta}_{n}=\tilde{\zeta}_{n}^{\prime}+\tilde{\zeta}_{n}^{\prime \prime}=\sum_{k=1}^{n} u_{k}^{\prime}+\sum_{k=1}^{n} u_{k}^{\prime \prime}$. Moreover, $\left(u_{k}^{\prime}\right)_{k \geq 1}$ is a martingale difference and for all $k \geq 2$, we have $\left|u_{k}^{\prime}\right| \leq 2 \frac{g^{\star}}{g_{\star}} q_{n}^{-1 / 4}$ and

$$
\mathbb{E}_{S}\left(\left(u_{k}^{\prime}\right)^{2} \mid \mathcal{F}_{k-1}\right)=\frac{1}{q_{n}} Q\left(\frac{x_{k}-z_{0}}{h}\right) \frac{g^{2}\left(x_{k}, S\right)}{g^{2}\left(z_{0}, S\right)} \operatorname{Var}\left(\xi_{1} \mathbb{I}_{\left|\xi_{1}\right| \leq q_{n}^{1 / 4}}\right) .
$$

Write

$\sum_{i=1}^{n} \mathbb{E}_{S}\left(\left(u_{i}^{\prime}\right)^{2} \mid \mathcal{F}_{i-1}\right)=\frac{\operatorname{Var}\left(\xi_{1} \mathbb{I}_{\left|\xi_{1}\right| \leq q_{n}^{1 / 4}}\right)}{q_{n}} \sum_{i=1}^{n} Q\left(\frac{x_{i}-z_{0}}{h}\right) \frac{g^{2}\left(x_{i}, S\right)}{g^{2}\left(z_{0}, S\right)}=\frac{G_{n}(S)}{q_{n}} a_{n}$,

where $G_{n}(S)=\sum_{i=1}^{n} Q\left(\frac{x_{i}-z_{0}}{h}\right) \frac{g^{2}\left(x_{i}, S\right)}{g^{2}\left(z_{0}, S\right)}$ and $a_{n}=\operatorname{Var}\left(\xi_{1} \mathbb{I}_{\left|\xi_{1}\right| \leq q_{n}^{1 / 4}}\right)$.

Denoting $r_{n}(S)=\frac{G_{n}(S)}{q_{n}} a_{n}$ and $\tau_{n}=\inf \left\{k: \sum_{i=1}^{k} \mathbb{E}_{S}\left(u_{i}^{\prime 2} \mid \mathcal{F}_{i-1}\right) \geq r_{n}(S)\right\}$, we obtain $\tau_{n}=\inf \left\{k: \sum_{i=1}^{k} Q\left(\frac{x_{i}-z_{0}}{h}\right) \geq q_{n}\right\}$ and $\tilde{\zeta}_{n}^{\prime}=\sum_{k=1}^{\tau_{n}} u_{k}^{\prime}$.

Let us show that $a_{n}$ and further $r_{n}(S)$ tend to 1 uniformly in $p \in \mathcal{P}_{\epsilon, L}$ and in $S \in \mathcal{U}_{z_{0}, \delta}$. Firstly we have:

$$
\begin{aligned}
\left|a_{n}-1\right| & =\left|\mathbb{E}\left(\xi_{1}^{2} \mathbb{I}_{\left|\xi_{1}\right| \leq q_{n}^{1 / 4}}\right)-\mathbb{E}\left(\xi_{1} \mathbb{I}_{\left|\xi_{1}\right| \leq q_{n}^{1 / 4}}\right)^{2}-1\right| \\
& \leq\left|\int_{-q_{n}^{1 / 4}}^{q_{n}^{1 / 4}} x^{2} p(x) d x-1\right|+\left|\int_{-q_{n}^{1 / 4}}^{q_{n}^{1 / 4}} x p(x) d x\right|^{2} .
\end{aligned}
$$

The Cauchy-Schwarz inequality brings us:

$$
\left|\int_{-q_{n}^{1 / 4}}^{q_{n}^{1 / 4}} x p(x) d x\right|^{2} \leq\left(\int_{-\infty}^{+\infty} x^{2} p(x) \mathbb{I}_{|x|>q_{n}^{1 / 4}} d x\right)\left(\int_{-\infty}^{+\infty} p(x) d x\right) \leq K_{p}\left(q_{n}^{1 / 4}\right) .
$$

Nevertheless by the definition of the set $\mathcal{P}_{\epsilon, L}$, we get

$$
\sup _{p \in \mathcal{P}_{\epsilon, L}} K_{p}(a):=\sup _{p \in \mathcal{P}_{\epsilon, L}} \int_{-\infty}^{+\infty} x^{2} \mathbb{I}_{|x|>a} p(x) d x \underset{a \rightarrow \infty}{\longrightarrow} 0 .
$$

From here it follows that

$$
\sup _{p \in \mathcal{P}_{\epsilon, L}} \sup _{S \in \mathcal{U}_{z_{0}, \delta}}\left|a_{n}-1\right| \leq 2 \sup _{p \in \mathcal{P}_{\epsilon, L}} K_{p}\left(q_{n}^{1 / 4}\right)
$$


so the left term goes to zero as $n$ goes to infinity.

Using assumption (4) and the inequality

$$
\left|r_{n}(S)-1\right| \leq\left|\frac{G_{n}(S)}{q_{n}}-1\right|+\frac{G_{n}(S)}{q_{n}}\left|a_{n}-1\right|
$$

we get the convergence of $r_{n}(S)$ to 1 uniformly in $p$ and in $S$.

Applying lemma A.4 shows on the one hand the convergence in distribution of $\zeta_{n}^{\prime}$ to $\mathcal{N}(0,1)$ uniformly in $p \in \mathcal{P}_{\epsilon, L}$ and in $S \in \mathcal{U}_{z_{0}, \delta}$ because the function $\rho$ in lemma A.4 does not depend on the law of the martingale difference. In fact, if $\Phi$ denotes the standard Gaussian distribution function, one has

$$
\begin{aligned}
& \left|\mathbb{P}\left(\sum_{k=1}^{\tau_{n}} u_{k}^{\prime} \leq x\right)-\Phi(x)\right| \\
\leq & \left|\mathbb{P}\left(\sum_{k=1}^{\tau_{n}} u_{k}^{\prime} \leq x\right)-\Phi\left(x / \sqrt{r_{n}(S)}\right)\right|+\left|\Phi(x)-\Phi\left(x / \sqrt{r_{n}(S)}\right)\right| .
\end{aligned}
$$

The second term of the right member of this inequality tends toward zero uniformly in $p$, in $S$ and in $x$ because $r_{n}(S) \rightarrow 1$ uniformly in $p$ and in $S$ and because $\Phi$ is uniformly continuous on $\mathbb{R}$.

On the other hand one has $\mathbb{E}\left|\tilde{\zeta}_{n}^{\prime \prime}\right| \rightarrow 0$ uniformly in $p$ and in $S$. Indeed one have immediately $\mathbb{E}\left(\tilde{\zeta}_{n}^{\prime \prime 2}\right)=\frac{G_{n}(S)}{q_{n}} K_{p}\left(q_{n}^{1 / 4}\right)$. Then (24) and the Cauchy-Schwarz inequality yield

$$
\sup _{p \in \mathcal{P}_{\epsilon, L}} \sup _{S \in \mathcal{U}_{z_{0}, \delta}} \mathbb{E}_{S}\left|\tilde{\zeta}_{n}^{\prime \prime}\right| \rightarrow 0
$$

Using Markov's inequality, we show that $\left(\tilde{\zeta}_{n}^{\prime \prime}\right)$ tends to 0 in probability uniformly in $p$ and in $S$.

As a consequence $\tilde{\zeta}_{n}=\tilde{\zeta}_{n}^{\prime}+\tilde{\zeta}_{n}^{\prime \prime}$ converges in distribution to $\eta \sim \mathcal{N}(0,1)$ uniformly in $p$ and in $S$. This immediately implies (23).

\section{A Appendix}

Lemma A.1 Fix $\nu \in] 0 ; \frac{1}{4}[$ and $\delta \in] 0 ; 1\left[\right.$. Then there exists an integer $n_{\nu, \delta}>0$ such that $S_{\nu} \in \mathcal{U}_{z_{0}, \delta}$ for all $n \geq n_{\nu, \delta}$.

Proof: First remark that $\int_{-1}^{1}\left(S_{\nu}\left(z_{0}+u h\right)-S_{\nu}\left(z_{0}\right)\right) d u=0$. Moreover one 
has

$$
\left|S_{\nu}^{\prime}(x)\right|=\frac{1}{\varphi_{n} h}\left|V_{\nu}^{\prime}\left(\frac{x-z_{0}}{h}\right)\right| \leq \frac{2\left\|l^{\prime}\right\|_{\infty}}{\nu^{2}} n^{\frac{-\beta+1}{2 \beta+1}}
$$

For any fixed $\delta$ in $] 0 ; 1[$, if we choose $n \geq 1$ such that

$$
n^{\frac{-\beta+1}{2 \beta+1}} \frac{2\left\|l^{\prime}\right\|_{\infty}}{\nu^{2}} \leq \delta^{-1} \text { i.e. } n \geq\left(\frac{2\left\|l^{\prime}\right\|_{\infty} \delta}{\nu^{2}}\right)^{\frac{2 \beta+1}{\beta-1}}
$$

then $S_{\nu} \in \mathcal{U}_{z_{0}, \delta}$.

Therefore we have the desired result.

Lemma A.2 We have the following limit:

$$
\varsigma_{n}^{2} \underset{n \rightarrow \infty}{\longrightarrow} \int_{-1}^{1} \frac{V_{\nu}^{2}(z)}{g^{2}\left(z_{0}, 0\right)} d z .
$$

Proof: For sufficiently large $n$ we have

$$
\begin{aligned}
& \varsigma_{n}^{2}=\frac{1}{n h} \sum_{k=1}^{n} \frac{V_{\nu}^{2}\left(\frac{x_{k}-z_{0}}{h}\right)}{g^{2}\left(x_{k}, S_{\nu, u}\right)}=\frac{1}{h} \int_{z_{0}-h}^{z_{0}+h} \frac{V_{\nu}^{2}\left(\frac{x-z_{0}}{h}\right)}{g^{2}\left(x, S_{\nu, u}\right)} \mu_{n}(d x)=\int_{0}^{1} \frac{V_{\nu}^{2}\left(\frac{x-z_{0}}{h}\right)}{g^{2}\left(x, S_{\nu, u}\right)} \nu_{n}(d x) \\
& \text { with } \mu_{n}=\frac{1}{n} \sum_{k=1}^{n} \delta_{k / n}=\text { and } \nu_{n}=\frac{\mathbb{I}_{\left[z_{0}-h ; z_{0}+h\right]}}{h} \mu_{n} .
\end{aligned}
$$

Using hypothesis (6) and (7) to the function $g$, we can write for all $x \in[0 ; 1]$

$$
\begin{aligned}
\left|\frac{1}{g^{2}\left(x, S_{\nu, u}\right)}-\frac{1}{g^{2}(x, 0)}\right| & \leq \frac{1}{g_{\star}^{4}} \mid 2 g(x, 0) L_{x, 0}\left(S_{\nu, u}\right)+L_{x, 0}^{2}\left(S_{\nu, u}\right)+\Gamma_{x, 0}^{2}\left(S_{\nu, u}\right) \\
& +2 g(x, 0) \Gamma_{x, 0}\left(S_{\nu, u}\right)+2 L_{x, 0}\left(S_{\nu, u}\right) \Gamma_{x, 0}\left(S_{\nu, u}\right) \mid \\
& \leq \frac{1}{g_{\star}^{4}}\left(2 g^{\star} C_{0}\left\|S_{\nu, u}\right\|+C_{0}^{2}\left\|S_{\nu, u}\right\|^{2}+\left|\Gamma_{x, 0}\left(S_{\nu, u}\right)\right|^{2}\right. \\
& \left.+2 g^{\star}\left|\Gamma_{x, 0}\left(S_{\nu, u}\right)\right|+2 C_{0}\left\|S_{\nu, u}\right\|\left|\Gamma_{x, 0}\left(S_{\nu, u}\right)\right|\right) .
\end{aligned}
$$

Hence

$$
\begin{aligned}
& \left|\int_{0}^{1}\left(\frac{1}{g^{2}\left(x, S_{\nu, u}\right)}-\frac{1}{g^{2}(x, 0)}\right) \nu_{n}(d x)\right| \\
\leq & \frac{\left\|S_{\nu, u}\right\|}{g_{\star}^{4}} \int_{0}^{1} \nu_{n}(d x)\left(2 g^{\star} C_{0}+C_{0}^{2}\left\|S_{\nu, u}\right\|+\left(\sup _{x \in[0 ; 1]} \frac{\left|\Gamma_{x, 0}\left(S_{\nu, u}\right)\right|}{\left\|S_{\nu, u}\right\|}\right)^{2}\left\|S_{\nu, u}\right\|\right. \\
+ & \left.2 g^{\star}\left(\sup _{x \in[0 ; 1]} \frac{\left|\Gamma_{x, 0}\left(S_{\nu, u}\right)\right|}{\left\|S_{\nu, u}\right\|}\right)+2 C_{0}\left(\sup _{x \in[0 ; 1]} \frac{\left|\Gamma_{x, 0}\left(S_{\nu, u}\right)\right|}{\left\|S_{\nu, u}\right\|}\right)\left\|S_{\nu, u}\right\|\right) .
\end{aligned}
$$


As $\left(\nu_{n}\right)$ weakly tends to $2 \delta_{z_{0}}$ when $n \rightarrow \infty$, one has

$$
\lim _{n \rightarrow \infty} \int_{0}^{1} \nu_{n}(d x)=2 \text { et } \lim _{n \rightarrow \infty} \int_{0}^{1}\left(\frac{1}{g^{2}(x, 0)}-\frac{1}{g^{2}\left(z_{0}, 0\right)}\right) \nu_{n}(d x)=0 .
$$

Then taking into account hypothesis (8) and because $\left\|S_{\nu, u}\right\|$ tends to 0 as $n \rightarrow \infty$, we obtain on the one hand

$$
\int_{0}^{1}\left(\frac{1}{g^{2}\left(x, S_{\nu, u}\right)}-\frac{1}{g^{2}\left(z_{0}, 0\right)}\right) \nu_{n}(d x) \underset{n \rightarrow \infty}{\longrightarrow} 0
$$

On the other hand

$$
\int_{0}^{1} \frac{V_{\nu}^{2}\left(\frac{x-z_{0}}{h}\right)}{g^{2}\left(z_{0}, 0\right)} \nu_{n}(d x)=\frac{1}{n h} \sum_{k=1}^{n} \frac{V_{\nu}^{2}\left(\frac{x_{k}-z_{0}}{h}\right)}{g^{2}\left(z_{0}, 0\right)} \underset{n \rightarrow \infty}{\longrightarrow} \int_{-1}^{1} \frac{V_{\nu}^{2}(y)}{g^{2}\left(z_{0}, 0\right)} d y .
$$

Now, if $V_{\nu}^{\star}$ denotes the maximum of $V_{\nu}^{2}$ on $\mathbb{R}$, one has

$$
\begin{aligned}
\left|\varsigma_{n}^{2}-\int_{-1}^{1} \frac{V_{\nu}^{2}(z)}{g^{2}\left(z_{0}, 0\right)} d z\right| & \leq\left|\int_{0}^{1} \frac{V_{\nu}^{2}\left(\frac{x-z_{0}}{h}\right)}{g^{2}\left(x, S_{\nu, u}\right)} \nu_{n}(d x)-\int_{0}^{1} \frac{V_{\nu}^{2}\left(\frac{x-z_{0}}{h}\right)}{g^{2}\left(z_{0}, 0\right)} \nu_{n}(d x)\right| \\
& +\left|\int_{0}^{1} \frac{V_{\nu}^{2}\left(\frac{x-z_{0}}{h}\right)}{g^{2}\left(z_{0}, 0\right)} \nu_{n}(d x)-\int_{-1}^{1} \frac{V_{\nu}^{2}(z)}{g^{2}\left(z_{0}, 0\right)} d z\right| \\
& \leq V_{\nu}^{\star} \int_{0}^{1}\left|\frac{1}{g^{2}\left(x, S_{\nu, u}\right)}-\frac{1}{g^{2}\left(z_{0}, 0\right)}\right| \nu_{n}(d x) \\
& +\left|\int_{0}^{1} \frac{V_{\nu}^{2}\left(\frac{x-z_{0}}{h}\right)}{g^{2}\left(z_{0}, 0\right)} \nu_{n}(d x)-\int_{-1}^{1} \frac{V_{\nu}^{2}(z)}{g^{2}\left(z_{0}, 0\right)} d z\right| .
\end{aligned}
$$

Let $n$ goes to $\infty$ and then we have completed the proof of lemma A.2.

Lemma A.3 The variance $\sigma_{n}^{2}(S)$ of $\zeta_{n}$ satisfies

$$
\sigma_{n}^{2}(S) \underset{n \rightarrow \infty}{\longrightarrow} g^{2}\left(z_{0}, S\right) \text {. }
$$

Proof: One has

$$
\sum_{k=1}^{n} Q\left(\frac{x_{k}-z_{0}}{h}\right) g^{2}\left(x_{k}, S\right)=n \int_{z_{0}-h}^{z_{0}+h} g^{2}(x, S) \mu_{n}(d x)
$$

with the measure $\mu_{n}=\frac{1}{n} \sum_{k=1}^{n} \delta_{k / n}$.

We know that $\left(\mu_{n}\right)_{n \geq 1}$ weakly tends to the uniform measure on $[0 ; 1]$. 
Moreover for sufficiently large $n$,

$$
\frac{1}{n h} \sum_{k=1}^{n} Q\left(\frac{x_{k}-z_{0}}{h}\right) g^{2}\left(x_{k}, S\right)=\int_{z_{0}-h}^{z_{0}+h} g^{2}(x, S) \nu_{n}(d x)
$$

with $\nu_{n}=\frac{\mu_{n} \mathbb{I}_{\left[z_{0}-h ; z_{0}+h\right]}}{h}$.

Like this $\left(\nu_{n}\right)_{n \geq 1}$ weakly tends to $2 \delta_{z_{0}}$, the Dirac measure at $z_{0}$, when $n \rightarrow \infty$. Then we can conclude as we remember that $\frac{q_{n}}{\varphi_{n}^{2}} \underset{n \rightarrow \infty}{\longrightarrow} 2$ and that $n h=\varphi_{n}^{2}$.

Lemma A.4 (Freedman, 1971, pp. 90-91) Let $\delta \in] 0 ; 1[$ and $r>0$. Assume that $\left(u_{k}\right)_{k \geq 0}$ is a martingale difference with respect to the filtration $\left(\mathcal{F}_{k}\right)_{k \geq 0}$ such that $\left|u_{k}\right| \leq \delta$ for all $k$ and $\sum_{k=1}^{\infty} \mathbb{E}\left(u_{k}^{2} \mid \mathcal{F}_{k-1}\right) \geq r$.

Define $\tau=\inf \left\{n: \sum_{k=1}^{n} \mathbb{E}\left(u_{k}^{2} \mid \mathcal{F}_{k-1}\right) \geq r\right\}$.

Then there exists a function $\rho:] 0 ;+\infty[\rightarrow[0 ; 2]$ not depending on the distribution of the martingale difference, such that $\lim _{x \rightarrow 0} \rho(x)=0$ and

$$
\sup _{x \in \mathbb{R}}\left|\mathbb{P}\left(\sum_{k=1}^{\tau} u_{k} \leq x\right)-\Phi(x / \sqrt{r})\right| \leq \rho(\delta / \sqrt{r})
$$

where $\Phi$ is the standard Gaussian distribution function.

\section{References}

Barron, A., Birgé, L. and Massart, P. (1999), Risk bounds for model selection via penalization, Probab. Theory Related Fields 113, 301-413.

Billingsley, P. (1999), Convergence of probability measures, Wiley Series in Probability and Statistics: Probability and Statistics, John Wiley \& Sons Inc., New York, A Wiley-Interscience Publication.

Dahlhaus, R. (1995), Efficient location and regression estimation for long range dependent regression models, Ann. Statist. 23, 1029-1047.

Donoho, D.L. (1994), Asymptotic minimax risk for sup-norm loss: solution via optimal recovery, Probab. Theory Related Fields 99(2), 145-170.

Donoho, D.L. (1994), Statistical estimation and optimal recovery, Ann. Statist. $22,238-270$.

Donoho, D.L., Johnstone, I.M., Kerkyacharian, G. and Picard, D. (1995), Wavelet shrinkage: asymptopia?, J. Roy. Statist. Soc. Ser. B 57, 301-369.

Donoho, D.L. and Liu, R.C. (1991), Geometrizing rates of convergence. III, Ann. Statist. 19, 668-701.

Efromovich, S. (1999), Nonparametric Curve Estimation. Methods, Theory and Applications, Springer, Berlin, New York. 
Efromovich, S. (2007), Sequential design and estimation in heteroscedastic nonparametric regression, Sequential Anal. 26(1), 3-25.

Efromovich, S. and Pinsker, M. (1996), Sharp-optimal and adaptive estimation for heteroscedastic nonparametric regression, Statist. Sinica 6(4), 925-942.

Freedman, D. (1971), Brownian Motion and Diffusion, Holden Day, San Francisco.

Galtchouk, L. and Pergamenshchikov, S. (2006), Asymptotically efficient estimates for nonparametric regression models, Statist. Probab. Lett. 76, 852860.

Galtchouk, L. and Pergamenshchikov, S. (2006), Efficient adaptive nonparametric estimation in heteroscedastic regression models, Preprint of the Strasbourg Louis Pasteur University, IRMA, available online at http:/ hal.archives-ouvertes.fr/hal-00129707/fr/

Goldfeld, S. and Quandt, R. (1972), Nonlinear Methods in Econometrics, North-Holland, Amsterdam, London.

Golubev, G.K. (1992), Asymptotically minimax estimation of a regression function in an additive model, Problems Inform. Trans. 28, 3-15.

Golubev, G.K. and Nussbaum, M. (1993), Adaptive spline estimates in a non parametric regression model, Theory Probab. Appl. 37 521-529.

Gunst, R.F. and Mason, R.L. (1980), Regression Analysis and its Applications: A Data oriented Approach, Marcel Dekker, New York.

Ibragimov, I.A. and Has'minskii, R.Z. (1981), Statistical Estimation: Asymptotic Theory, Springer, Berlin, New York.

Kalifa, J. and Mallat, S. (2003), Thresholding estimators for linear inverse problems and deconvolutions, Ann. Statist. 31, 58-109.

Korostelev, A. (1993), Exact asymptotically minimax estimator for nonparametric regression in uniform norm, Theory Probab. Appl. 38, 737-743.

Nemirovski, A. (2000), Topics in non-parametric statistics, in: Lectures on probability theory and statistics (Saint-Flour, 1998), Vol. 1738 of Lecture Notes in Math., Springer, Berlin, pp. 85-277.

Nussbaum, M. (1985), Spline smoothing in regression models and asymptotic efficiency in $L_{2}$, Ann. Statist. 13, 984-997.

Sacks, J. and Strawderman, W. (1982), Improvements on linear minimax estimates, in: Statistical decision theory and related topics, III, Vol. 2 (West Lafayette, Ind., 1981), Academic Press, New York, pp. 287-304.

Sacks, J. and Ylvisaker, D. (1981), Asymptotically optimum kernels for density estimation at a point, Ann. Statist. 9(2), 334-346. 